\title{
La última baja de la División Azul (de la metralla al hepatocarcinoma)
}

\author{
Lisbona Gil A. ${ }^{1}$
}

Sanid. mil. 2012; 68 (2): 119-122; ISSN: 1887-8571

\section{RESUMEN}

Presentamos aquí el caso clínico y evolución de un cabo de la División Azul, que habiendo sido herido en el frente ruso, sector de Nitlikino, por metralla, el 5 de diciembre de 1941, falleció cincuenta y un años más tarde como consecuencia probablemente de dicha herida.

The last casualty of 250 Wehrmacht Division (Blue Division) (from shrapnel to liver carcinoma) SUMMARY

We present the case of a corporal belonging to 250 Wehrmacht Division (Blau Division), that after he was hurt by shrapnel in the Russia front during the II World War, fifth of December 1941, he died fifty one years later, owing probably to that wound.

\section{ANTECEDENTES HISTÓRICOS}

El Subsector de Nitlikino, situado en el flanco izquierdo del avance iniciado por la División en dirección Sur, y por el margen Este del río Wolchow, era -dadas las condiciones del terreno y despliegue de las tropas- el punto elegido por el enemigo en sus continuos ataques, que se veían favorecidos por la ventaja de la sorpresa en un terreno de bosque que rodeaba el pueblo y permitía el fácil y posible envolvimiento de la posición. Mandaba este Subsector el comandante Suárez Roselló (III Batallón, Regimiento 263).

El 2 de noviembre de 1941 el Ejército Ruso ataca Nitlikino continuando los ataques hasta el día 14 del mismo mes, quedando tan quebrantado que cesa en su empeño durante quince días, sin dejar por ello de hostigar constantemente la posición con armas pesadas. Del 1 al 3 de diciembre el enemigo reanuda los ataques, ejecutando golpes de mano que son rechazados. El día 3 de diciembre la Artillería, morteros pesados, antitanques y armas automáticas, efectúan intenso fuego de hostigamiento sobre Nitlikino, seguido de un asalto que es rechazado.

El día 5 de diciembre las 13 horas sale el enemigo de sus posiciones y es detenido a los 400 metros; a las 17 es detenido a los 300 metros y es pegado al terreno; a las 18,15 avanza nuevamente y se les detiene a los 200 metros; a las 23,30, después de otro intenso fuego de Artillería, morteros pesados y antitanques, logra apoderarse de una avanzadilla al Sureste del pueblo tras haber sido muertos o heridos todos sus defensores. Con las escasas reservas de que aún se disponía - dos pelotones al mando de un oficial- se reconquista la posición perdida.

Reitera sus ataques el enemigo el día 6, haciéndole fracasar en su intento el fuego de nuestras posiciones. El 8 de diciembre -en cumplimiento de órdenes superiores - se efectúa el repliegue del Subsector de Nitlikino. Los combates se desarrollaron con temperaturas de 30 y $35^{\circ}$ bajo cero y una capa de nieve de 70 centímetros. Se produjeron 109 muertos, 258 heridos y 103 congelados, es decir 470 bajas que representan más del $50 \%$ de los efectivos del Batallón. Se le hicieron al enemigo más de 1000 muertos y 300 prisioneros, capturándole abundante material de guerra y armamento de toda clase ${ }^{1}$.

\section{EVOLUCIÓN DEL HERIDO}

Fue evacuado el 6-12-41 al Puesto de Socorro de la División de Grigorowo (Rusia); el 11-12-41 al Hospital de Sorchi (Rusia); el 19-12-41 al Hospital (español) de Porchow (Rusia) y 1-1-42 al Hospital de Bromberg (Polonia) donde permaneció hasta el 9-4-42 en que es evacuado al Hospital de Friedrichshagen (Alemania). El 18-4-42 ingresa en el Hospital de Hof (Alemania), el 23-4-42 en el Hospital del Generalísimo (San Sebastián) y el 254-42 en el Hospital Militar Vista Alegre (Madrid), siendo dado de alta el 25-12-1942.

Por su interés, se transcribe a continuación la Hoja Clínica (literal con los errores) durante su estancia en el Hospital de Bromberg (Polonia)

${ }^{1}$ Tcol. Médico. Hospital Central de la Defensa Gómez Ulla. Servicio de Endocrinología y Nutrición. Madrid. España.

Dirección para correspondencia: Dr. Lisbona Gil. Servicio de Endocrinología y Nutrición. Hospital Central de la Defensa Gómez Ulla. Glorieta del Ejército 1. 28047 Madrid. España. Email: alisgi@oc.mde.es

Recibido: 7 de diciembre de 2011

Aceptado: 23 de marzo de 2012 
Hospital de Reserva (Reserve-Lazarett) BROMBERG. (Departamento de los Heridos)

\section{Ejercito Sala $4^{\circ}$ - 320 - 15997 - E \\ Hoja del Enfermeda (Español)}

Herida: 1.) Herida casco de granada - en asentaderas.

2.) Casco de granada - izquierda - vajo torax. Numero del enfermedad: $31 \mathrm{~b}$
Nombre: Germán

Apellido: Sacristán Vesperinas

Empleo: Cabo

Unidad: $263-3^{\circ}-12$

Nacido: 19.1.1918

Nombre de población: Aguilera

Provincia: Soria

Entrada en servicio: 2.7.41

Religion: Católica

Familiar: Padre, Mateo Sacristán

Domicilio: Aguilera - Soria salido el 14.12.41 de Tschudowo.

el 18.12.41 con tren ambulancia Hospital de Reserva Bromberg.

al 8.1.42 ingreso Departamento de los enfermos No. II b.

al 9.4.42 por causa de convalecencia Berlin Friedrichshagen.

dado de alta: 17.4.42. Inútil para el servicio.

a donde: de Berlin a España.

\section{HOJA CLÍNICA}

A. Al dia 6.12.1941 a consecuencia de herida por casco de granado al lado izquierdo de pecho y al lado izquierdo del Glutäal. De resulta de herida tiene vómitos de sangre.

B. Al lado izquierdo del pecho casi a cuatro cmtr. del teta el orificio del casco es en forma de céntimo.

La respiracion es debil y poco pulso.

1. En el lado izqu. del Glutäal una herida de un tamaño un poco mas grande que el anterior. Los alrededores ilesos.

2. Radiografia según anexo.

16.12.41. La temperatura sube hasta $38.4^{\circ}$. Infeccion gripal. El estado es bueno. Hay posibilidad de una infeccion tuberculosa. Traslado a otro hospital por faltar los medios para curarle. El jefe medico del departamento de heridos.

19.12.41. Ingresa en la Clinica cirurgia de esta $2^{\mathrm{a}}$ Estacion. Pasa a la $1^{\text {a }}$ de Cirurgia en el dia de la fecha.

$$
\text { El jefe de la Clinica }
$$

19.12.41I. Ingresa en esta Clinica de cirurgia procedente de la $2^{\text {a }}$ Estacion. Presenta N.m. en cadera izquierda con metralla incluida. Tiene neuralgias mediadas de Plexo sacro. Tiene metralla incluida en pulmon izquierdo.

1.1.42. Ingreso del tren Ambulancia en el Departamento de los Enfermos No II del Hospital de Reserva, Bromberg.

Al lado del pecho izq. aprox. a la altura de la $5^{\text {a }}$ costilla en la parte anterior presenta una herida superficial del tamaño aprox. de una moneda de 10 Pfennig. Las partes cercanas a la herida son dolorosas a la presión. Presenta una herida superficial del tamaño aprox. de una moneda de dos marcos en la parte superior y externa del cuadrante del trasero izqu.
En el 4 y 5 dedo del pie izquierdo en su parte bajo zonas heridas con pequeña tendencia a curación. Vendas das todas las heridas que fueron tratadas con pomada de aceite de higado de bacalao.

Al toser se queja de dolores en la parte del pecho. No se observa ningún síntoma de enfermedad en el pulmón.

5.1.42. Las heridas secrejan aun mucho. El estado general es malo. T. estado subferil. El enfermo se queja de tos y esputos. Se administran cataplasmas de pecho y Jodo-Kali. Los esputos fueron hasta ahora negativos a tuberculosis.

8.1.42. S. ha sido hoy trasladado a la IIb sección.

Bromberg, el 8 de enero de 1942.

El Jefe supremo de la sección IId

Dr. Wittchen. Teniente medico

\section{Hospital de Reserva. BROMBERG. Departamento de los heridos IIb}

8.1.42. S. ha tenido hoy entrada en éste departamento. La temperatura es aun subferil. Las heridas de la parte izqu. del pecho y de la nalga izqu. continuan segregando mucho. El entumecimiento de los dedos del pie izqu. son tratados con baños de pies y con pomada aceize de higado de bacalao. Los irritaciones han ido disminuyendo. El estado general malo.

12.1.42. La radiografia presenta irregularmente dentado en el parte bajo un casco de granada en forma de hueso de cueza.

19.1.42. La temperatura hacia $37^{\circ}$. Estado general malo. B.S.G. 30/50. Sputu por Tbc. negativo. Análisis de la sangre mira la curva. H.B. $84 \%$. El mutilado lamenta de fuertes dolores en el pecho izqu. El toser desde principio muy fuerte se ha suavizado. El apetito es bueno. Pulmon: regular. La respiración es absolutamente limpia y vesicular. El lado izqu. del pecho se queda atrás durante la respiración.

23.1.42. B.S.G. 14 / 26. Continua a lamentarse de dolores en el pecho izqu. Diagnostico de los pulmones normal. El estado general no esta mejor. Los heridos a lado izqu. del pecho en buena disposición.

5.2.42. La herida al lado izqu. del trasero ha continuado de empequeñaserse. Los dolores en el pecho se han aflojado.

17.2.42. La herida al lado izqu. del Glutaäus esta abierto todavia, solamente como un guisante y esta propria y no discanda. El paciente siga bien.

27.2.42. Radiografia del pulmon igual del 12.1 .42

2.3.42. La herida al trasero se ha cerrado. Abajo como el ancho de una mano se ha indicado en la misma anchura dolores de presion. Lamenta de dolores en el pecho y falta de apetito. El descenso del útero sa ha acelerado de modo que una radiografia se ha causada. Pero no resulta nada de especial. Paciente recibe Insulin para mejorar su estado general.

14.3.42. El apetito se ha mejorado apenas. Paciente se levanto. Hoy la temperatura va subiendo de nuevo hasta $38,4^{\circ}$. Lamenta de dolores al lado izqu. del pecho. Por lo demás se ha indicado dolores ligeras debajo de la costilla, nauseas ligeras y gusto amargo. Sensibilidad de presión cerca de la bilis se ha indicada. Diagnostico de la orina normal.

20.3.42. Hace pocos dias que la temperatura se ha ido subiendo hasta $38^{\circ}$, falta de apetito, eructa amargo y sensibilidad 


\section{La última baja de la División Azul (de la metralla al hepatocarcinoma)}

de presión cerca de la bilis. Recibe compresas encima del vientre, sal de Karlsbad y comida vegetariana. Despues los dolores van extinguiendose. En la orina ningun color de la bilis y analisis de la sangre $17 / 34$

27.3.42. Solamente muy pocos dolores, ninguna temperatura. Desayuno de prueba no da resulta ningun indicio para un trastorno del estómago.

4.4.42. Ningunos trastornos. Apetito y estado general son bien.

7.4.42. S. lamenta durante de la respiración profunda de dolores ligeras en el lado izqu. del pecho. Corazon y pulmon: sin diagnostico. En V dentro las costillas $2 \mathrm{~cm}$ medial de la linea axilar anterior una cicatriz, grande como el punto del meñique, aburrido. removiendo firme. Ancho de una mano debajo del intestinal al lado izqu. una herida grande como un guisante en una cavidad aproximadamente como un dedo de grueso con poca secreción.

\section{Diagnostico definitivo: Herida casco de granada - izqu.-bajo Torax - izqu. Glutäal}

\section{Resumen}

S. fué herido el 6.12.41 por casco de granada al Glutäal izqu. y al lado izqu. delantero del pecho. El 1.1.42 fue trasladado del tren ambulancia al Hospital de Reservas de Bromberg. Las heridas han curado sin complicaciones pero el estado general fué malo mientras varias semanas. Primeramente hacia sospecho que fué tuberculosis pulmonar pero no se ha confirmado. Durante algun tiempo se ha entregado Insulin contra la falta de apetito y el peso insuficiente. El estado general fué satisfactorio durante las ultimas semanas.

S. ha salido del casa de convalecencia de la Division Azul, Berlin - Friedrichshagen, Mueggelseedamm 92 el dia 9 de avril 1942 ha duracion de la estancia en la casa de convalecencia y la utilidad ulterior tiene que decidir el medico directivo.

Bromberg, el 8 de avril de 1.942

El jefe medico

Dr. Rehmers

Comandante medico

Bromberg, el 8 de avril de 1.942

El jefe supremo de la sección II B

Dr. Wittchen

Teniente medico

\section{Comentarios}

Durante su estancia en el Hospital de Bromberg cabe destacar por un lado, la sospecha de la existencia de una tuberculosis pulmonar que no llegó a ser demostrada y por otro lado la administración de insulina como hormona anabólica para mejorar el apetito y el estado general del herido.

Es evacuado a España, ingresando en el Hospital Militar de Vista Alegre el 25-4-42 y dado de alta el 25-12-42. Durante este tiempo fue diagnosticado de tuberculosis pulmonar y tratado con neumotórax terapéutico. En 1951 presentó un rebrote de la tuberculosis practicándosele en 1953 una lobectomía superior izquierda y un año más tarde, por nuevo episodio de hemoptisis, una toracoplastia izquierda. Precisó varias transfusiones de sangre durante y después de las intervenciones quirúrgicas.
En 1969 fue ingresado en el Hospital La Paz, por hematemesis secundaria a ulcus duodenal. Presentaba analíticamente, elevación de la glucemia y las transaminasas son descritas como normales. En 1971, es ingresado de nuevo por dolor en hipocondrio derecho, siendo diagnosticado de quiste hidatídico hepático calcificado siendo desestimada la intervención quirúrgica por insuficiencia ventilatoria restrictiva y por la probable inactividad de la hidatidosis. Analíticamente se observó elevación de la GOT (50 U/1) y es diagnosticado de diabetes. En 1974, por nuevo episodio de hemorragia digestiva se le practica una gastrectomía tipo Billroth I. Las transaminasas seguían elevadas. En ninguno de estos ingresos, consta que fueran necesarias transfusiones de sangre. En un nuevo ingreso en 1977 ya es diagnosticado de hepatopatía crónica difusa no filiada.

En 1985, acude a la consulta de Endocrinología de la Policlínica Naval "Nuestra Sra. del Carmen" para tratamiento, control y seguimiento de una diabetes tipo 2. Presentaba elevación de las transaminasas y la serología demostró ser positiva para el virus de la hepatitis B (VHB) y C (VHC). Una biopsia hepática demostró la existencia de una cirrosis hepática probablemente secundaria a VHB y/o VHC. El paciente no ingería alcohol desde hacía más de 30 años, a raíz de la elevación de las transaminasas y las concentraciones de hierro y ferritina fueron normales. Posteriormente, desarrolló un hepatocarcinoma que fue la causa última del fallecimiento.

De esta forma, consideramos, que a consecuencia del hacinamiento en campaña, la herida de metralla en pulmón izquierdo en diciembre de 1941 y el estado de extrema debilidad del herido, el cabo G.S.V. desarrolló una tuberculosis pulmonar en pulmón izquierdo, que precisó tratamiento con neumotórax terapéutico, lobectomía y toracoplastia, precisando varias transfusiones de sangre con contagio del VHB y VHC. Posteriormente desarrolló una hepatopatía crónica, cirrosis hepática y hepatocarcinoma, con fallecimiento en junio de1993, 51 años después de haber sido herido.

\section{RECONOCIMIENTO DEL GOBIERNO ALEMÁN DEL CABO G.S.V. COMO BAJA DE COMBATE}

Tras el fallecimiento, se iniciaron los trámites para obtener una pensión del Gobierno Alemán, para su viuda. El argumento era el ya expuesto anteriormente y fue redactado y enviado al Versorgungsamt Karlsruhe en los siguientes términos:

D. Germán Sacristán Vesperinas. Herido de metralla en hemitórax izquierdo el 06.12.1941 en el frente ruso, al otro lado del Volchow. Fue evacuado al Hospital de Bromberg donde se sospechó una infección tuberculosa, siendo los esputos negativos para bacilo de Koch.

Durante su estancia en el Hospital de Bromberg, fue tratado con insulina por anorexia y pérdida de peso, siendo dado de alta y enviado a España el 17-abril-1942. A su llegada a España desarrolló una tuberculosis pulmonar sobre el pulmón herido realizándose una toracoplastia izquierda, siendo transfundido durante la intervención quirúrgica. Como consecuencia de la transfusión de sangre o por la administración de insulina con jeringas no desechables (no existían en aquella época) se le contagió una hepatitis $B$ y $C$ que 
evolucionó a una hepatitis crónica y posterior cirrosis hepática, sobre la que se injertó un hepatocarcinoma que fue la causa de su fallecimiento.

Consideramos a éste, como un caso curioso y único de fallecimiento como consecuencia de una herida de metralla acaecida 51 años antes, siendo posiblemente la última baja de la DIVISIÓN AZUL.

Madrid 14-12-93

Dr. A. Lisbona Gil

Capitán Médico

Policlínica Naval N ${ }^{\mathrm{a}}$ Sra. del Carmen. Madrid

\section{Contestación del VERSORGUNGSAMT KARLSRUHE (literal)}

\section{Señor/Don \\ Dr. med. A.Lisbona Gil \\ Policlínica Naval}

Muy estimado señor Colega

Con fecha de 14-12-93 Ud. nos remitió un informe médico sobre el paciente precitado. En esto Ud. tiene la comprensión, que el precitado mutilado de la guerra tenía relación con el tratamiento médico como consecuencia de su sufrimiento de los servicios militares una transfusión de una hepatitis B y C, cual entonces ha llevado al muerte por la cirrosis hepática y hepatitis crónica.

Para la apreciación de los derechos provisionales, le ruego a Usted por lo posible a una contestación más exacta a las preguntas siguientes:

1. ¿Desde cuando y cuanto tiempo se lo trataban el Sr. Sacristán Vesperinas en la clínica suya?

2. ¿Las infecciones de hepatitis B y hepatitis C se lo sospecharon solamente o se les aseguraron serológico? ¿Cuáles exactos síntomas serológicos se lo habían hallado y al cuál tiempo esto fue?

3. ¿Usted podría informarnos más exacto sobre la anamnesis, en especial a lo que, si el sr. Sacristán Vesperinas desde final de guerra también ha sufrido con las enfermedades otras o tenía accidentes y él en este margen ha percibido p.e. transfusiones de la sangre? Por favor, también indíquenos, ¿si a Vd. están conocidos las costumbres de beber (vino, cerveza, aguardiente, etc) y por cuál tiempo el habia tomado alcohol en cantidades importantes?

4. Ademas le ruego a Vd. enviarnos fotocopia por cuanto ello sera posible, en especial los signos laboratorios. Si esto sería posible, le agradeceria mucho por remitirnos completa historia de enfermedades. Pero, esto tenía que pasar por el "CERTIFICADO".

Nosotros esta estoria de la enfermedad se la devolveremos también como carta certificada.

5. ¿Se puede notificar de sus documentos que el sr. Sacristán Vesperinas también fue en tratamiento en las clinicas otras? En este caso, Vd. también tendría que informarnos sobre esto.
La indemnización de los gastos se resuelta según las costumbres del país en especial. Se lo ruega remitirnos el dictamen en simple y la liquidación en doble producción.

Con saludos muy atentementes. Karlsruhe 24-05-1994. Fdo. Dr. Resch

\section{La contestación fue la siguiente}

Estimado colega:

Le envío el informe del Divisionario Azul, Don Germán Sacristán Vesperinas, donde se detallan exhaustivamente sus antecedentes patológicos. Asimismo le envío una fotocopia de la serología de hepatitis, realizada en nuestro hospital.

Desde un punto de vista estrictamente médico, el único nexo de unión entre la herida de metralla y su muerte, 51 años más tarde, es el desarrollo de una tuberculosis pulmonar sobre el pulmón herido, quizás por asentar esta infección en la zona de dicho pulmón mal ventilado; o quizás por haberla adquirido debido al estado de debilidad por las heridas recibidas. Por supuesto, el contagio del virus de la hepatitis B y C se realizó en España cuando tuvo que ser intervenido de dicho pulmón.

Desde un punto de vista romántico, me apetece pensar que D. Germán Sacristán fue la última baja de la 250 División.

Le ruego disculpe la demora en contestar pero no ha sido muy fácil reconstruir toda la historia patológica.

Un afectuoso saludo. Dr. A. Lisbona Gil

La respuesta inmediata a esta última carta fue la concesión a su viuda de una pensión, reconociendo que el fallecimiento de G.S.V., había ocurrido 51 años más tarde a consecuencia de una herida de combate.

\section{BIBLIOGRAFÍA}

1. R. Suárez Roselló. Esto es..jHISTORIA!. Blau División. Boletín de la Hermandad Provincial de la División Azul. Mayo 1989. No 358

\section{OBSERVACIONES}

1. Dado que el artículo es de Historia y Humanidades, como homenaje al cabo herido y el interés del documento para la Asociación de Divisionarios figura en el mismo nombre y datos personales del mismo.

2. Dado que se han mantenido textualmente partes de los informes médicos alemanes originales, algunas palabras y términos con errores ortográficos o de otro tipo, estos figuran en letra cursiva. 